\title{
Numerical Simulation of 3D Boundary-Driven Acoustic Streaming in Microfluidic Devices ${ }^{\dagger}$
}

\author{
Junjun Lei, Martyn Hill", and Peter Glynne-Jones \\ Faculty of Engineering and the Environment, University of Southampton, Southampton, UK \\ m.hill@soton.ac.uk. \\ †Electronic Supplementary Information (ESI) is available. 3D, top and side views of the \\ simulated trajectories of $0.5 \mu \mathrm{m}$ particles are included.
}

This article discusses three-dimensional (3D) boundary-driven streaming in acoustofluidic devices. Firstly, the 3D Rayleigh streaming pattern in a microchannel is simulated and its effect on the movement of microparticles of various sizes is demonstrated. The results obtained from this model show good comparisons with 3D experimental visualisations and demonstrate the fully 3D nature of the acoustic streaming field and the associated acoustophoretic motion of microparticles in acoustofluidic devices. This method is then applied to another acoustofluidic device in order to gain insights into an unusual in-plane streaming pattern. The origin of this streaming has not been fully described and its characteristics cannot be explained from the classical theory of Rayleigh streaming. The simulated in-plane streaming pattern was in good agreement with the experimental visualisation. The mechanism behind it is shown to be related to the active sound intensity field, which supports our previous findings on the mechanism of the in-plane acoustic streaming pattern visualised and modelled in a thin-layered capillary device.

\section{Introduction}

Particle manipulation using ultrasonic standing waves has gained increased attention in recent years as it is efficient and non-invasive. During the process of manipulation, acoustic streaming is typically found in addition to the acoustic radiation forces. In acoustofluidic particle manipulation devices, the acoustic streaming field is generally dominated by boundary-driven streaming, which is a result of the interaction between the acoustic oscillation and solid boundaries. Rayleigh ${ }^{1}$ was the first to present a theoretical analysis of a boundary layer driven acoustic streaming field. His solution only describes the fluid motion outside the viscous boundary layer, so it is commonly referred to as 'outer streaming' as well as 'Rayleigh streaming'. Subsequently, modifications to Rayleigh's solution have been proposed, most notably by Westervelt ${ }^{2}$, Nyborg $^{3}$ and Schlichting ${ }^{4}$, reviewed by Boluriaan et al. ${ }^{5}$ and Wiklund et al. ${ }^{6}$. Hamilton et al. ${ }^{7}$ derived an analytical solution for the acoustic streaming generated by a standing wave confined by parallel plates that solved the streaming field both inside and outside the viscous boundary layer. Another kind of boundary-driven streaming is transducer-plane streaming. Different from the better known classical streaming pattern, e.g. Rayleigh streaming ${ }^{1}$ and Eckart streaming ${ }^{8}$, whose vortex plane is normally perpendicular to the transducer face, the circulation of transducer-plane streaming is parallel to the transducer face. Such streaming patterns are typically generated in planar microfluidic resonators where the acoustic energy gradients in the lateral directions parallel to the transducer face are significant in addition to the gradients perpendicular to the transducer face ${ }^{9,10}$. The mechanism behind the transducer-plane streaming pattern was recently analysed and shown to be related to the acoustic intensity field ${ }^{9}$.

These theoretical analyses have been complemented by experimental work in acoustofluidic systems and numerical simulations. On the one hand, acoustic streaming and acoustophoretic motion of microparticles in acoustofludic devices have been measured using various methods, most notably micro particle image velocimetry $(\mu \mathrm{PIV})$ and particle tracking velocimetry (PTV). Experimental investigations have shown that $\mu \mathrm{PIV}^{11-13}$ and $\mathrm{PTV}^{14}$ ${ }^{15}$ are powerful tools for analysing 2D microchannel acoustophoresis. Fully 3D particle tracking has been demonstrated using $\mu \mathrm{PIV}$ with depth of correlations ${ }^{16}$ and astigmatism particle tracking velocimetry ${ }^{17-19}$. On the other hand, numerical simulations of acoustophoretic motion of microparticles can provide efficient prediction of experiments and provide effective guidance and optimization on the design of acoustofluidic devices to enhance or improve experiments. Many existing models of acoustic streaming simulation are based on 2D simplifications that consider only a cross-sectional area of the fluid chamber due to the high computational demand of 3D simulations. In these models, the acoustic field in the fluid layer is generally assumed to have a periodic distribution of constant amplitude as it is obtained from a uniform distribution of boundary vibration ${ }^{20-22}$. However, in real acoustofluidic devices, the acoustic field generated from the transducer does not always have a perfectly uniform distribution 
along the channel axis due to lateral modes, structural modes, transducer inhomogeneities and acoustic absorption at the ends of channels. Therefore, results obtained from simplified 2D models cannot fully represent real acoustofluidic devices and 3D models are necessary to provide better understanding and prediction of experiments. Recently, Lei et al. ${ }^{9}$ successfully simulated the transducer-plane streaming in a glass capillary by considering a 3D model using the computationally efficient limiting velocity finite element method.

In this paper we apply the limiting velocity finite element method to calculate the driving boundary conditions on a 3D fluid volume. We model two acoustofluidic devices described in the literature:

a) An acoustofluidic device investigated experimentally by Muller et al. ${ }^{23}$. Our simulated results are shown to be in good agreement with the experimental observations and provide evidence of 3D characteristics.

b) The second device was first presented by Hagsater et al. ${ }^{13}$. It was shown to present an unusual pattern of $6 \times 6$ in-plane streaming vortices that differed from that predicted by consideration of the Rayleigh streaming pattern and has not previously been explained. By modelling it here we are able to make suggestions as to the cause of this phenomenon.

In Section II, the numerical method used to simulate the acoustic streaming field in the main fluid is introduced. Then, the 3D Rayleigh streaming pattern in the first device is simulated and analysed in Section III, where the model, results and a discussion are presented. In Section IV, the unusual acoustic streaming pattern visualised in the second device is investigated and brief conclusions are drawn in Section V.

This paper demonstrates how 3D models add to our understanding of the streaming behaviours found in experimental devices. While many systems can be modelled appropriately with suitable 2D approximations, making the correct approximation a-priori is not always straightforward, and can only be judged accurate in hindsight from a 3D representation (be that a model or experimental results). For example in this paper (Section IV), a 2D model is not sufficient: streaming is driven by a boundary that is parallel to the plane of the observed streaming pattern.

\section{Numerical Method}

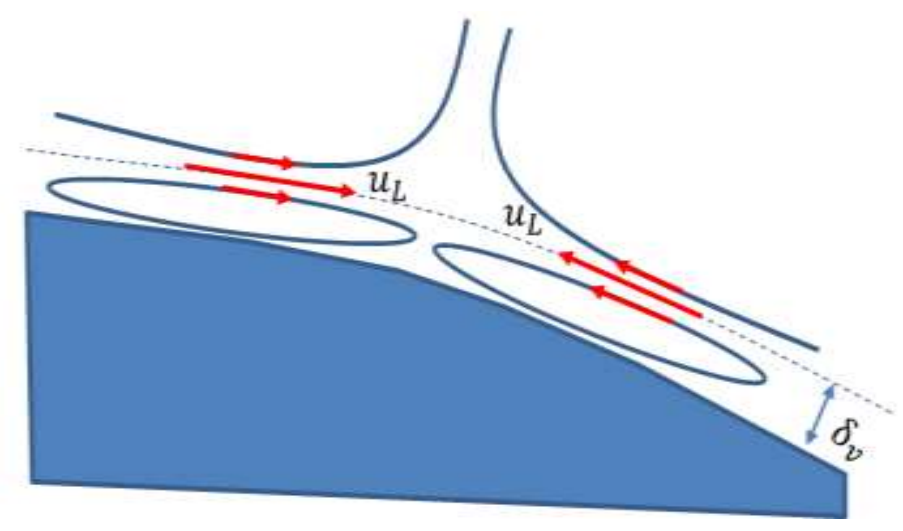

Fig. 1 Schematic of the limiting velocities over a solid surface, where $u_{L}$ is the limiting velocity and $\delta_{v}$ is the viscous penetration depth. Reprinted from Ref. ${ }^{\text {. }}$

Fig. 1 shows the schematic of the limiting velocity method we use to simulate the streaming field which is based on the analytical solutions first introduced by Nyborg ${ }^{3}$ and later modified by Lee and Wang ${ }^{24}$. This method decomposes the problem into three steps: (a) a linear acoustic model predicts the first order acoustic fields; (b) The limiting velocity is calculated at all boundaries as a function of the first order acoustic fields (essentially the streaming is driven by the interaction of the acoustic field with these boundaries); (c) A creeping flow model is used to calculate the resulting streaming flows. The limiting velocity only predicts the streaming field outside the viscous boundary layer, removing the need for a boundary-layer mesh and hence reducing computational load to the point where a 3D model is viable. We previously ${ }^{9}$ verified a 2D version of this method against Rayleigh's analytical solution $^{5}$ and used a 3D version to model and explain unexpected vortex patterns in the plane of the transducer in planar devices. This method is generally applicable to acoustofluidic devices working at $\mathrm{MHz}$ 
frequencies where the thickness of viscous boundary layer, described as $\delta_{v}$ in Fig. 1, is typically several orders of magnitude smaller than the dimensions of the fluid chamber so that only the streaming field outside the viscous boundary layer is of interest. The finite element package $\mathrm{COMSOL}^{25}$ was used to implement each of these steps, described in more detail below.

The first-order acoustic fields within the devices are simulated using COMSOL's 'pressure acoustic' physics, which solves the harmonic, linearized acoustic problem and takes the form:

$$
\nabla^{2} p=-\frac{\omega^{2}}{c^{2}} p
$$

where $\omega$ is the angular frequency, $c$ is the sound speed, and $p$ is the complex pressure defined at position $r$ using the relation,

$$
p_{t}(r, t)=\operatorname{Re}\left[p(r) e^{-i \omega t}\right]
$$

On a planar surface normal to $z$, the limiting velocity equations can be written as 9

$$
\begin{gathered}
u_{L}=-\frac{1}{4 \omega} \operatorname{Re}\left\{q_{x}+u_{1}^{*}\left[(2+i)\left(\frac{d u_{1}}{d x}+\frac{d v_{1}}{d y}+\frac{d w_{1}}{d z}\right)-(2+3 i) \frac{d w_{1}}{d z}\right]\right\}, \\
v_{L}=-\frac{1}{4 \omega} \operatorname{Re}\left\{q_{y}+v_{1}^{*}\left[(2+i)\left(\frac{d u_{1}}{d x}+\frac{d v_{1}}{d y}+\frac{d w_{1}}{d z}\right)-(2+3 i) \frac{d w_{1}}{d z}\right]\right\}, \\
q_{x}=u_{1} \frac{d u_{1}^{*}}{d x}+v_{1} \frac{d u_{1}^{*}}{d y}, \\
q_{y}=u_{1} \frac{d v_{1}^{*}}{d x}+v_{1} \frac{d v_{1}^{*}}{d y},
\end{gathered}
$$

where $u_{L}$ and $v_{L}$ are the two components of the limiting velocities over a vibrating surface, $\omega$ is the angular frequency, $u_{1}, v_{1}$ and $w_{1}$ are the three components of acoustic velocities along coordinates $x, y$ and $z, i=\sqrt{-1}$, and the superscript, *, denotes the conjugate value of the complex acoustic velocity.

COMSOL's 'creeping flow' physics was used to simulate the second-order acoustic streaming fields. This approximates the fluid as incompressible, and neglects inertial terms (Stokes flow) as the Reynolds numbers are much smaller than one in the devices presented in this paper. The governing equations for the streaming velocity field, $\boldsymbol{u}_{2}$, and associated pressure field, $p_{2}$, are

$$
\begin{gathered}
\nabla p_{2}=\mu \nabla^{2} \boldsymbol{u}_{2}, \\
\nabla . \boldsymbol{u}_{2}=0 .
\end{gathered}
$$




\section{Verification of the 3D streaming model within an acoustofluidic device}

1. Model configuration and Results

(a)

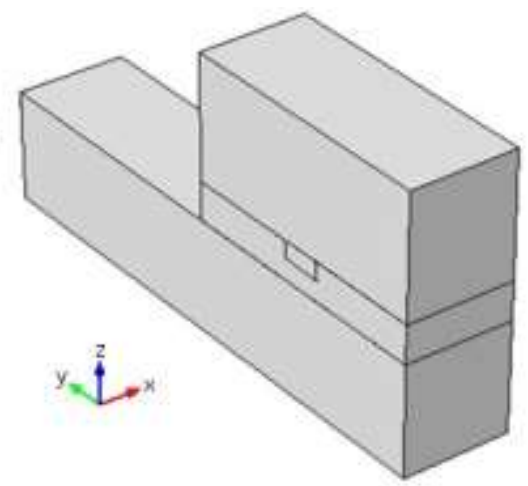

252

(b)

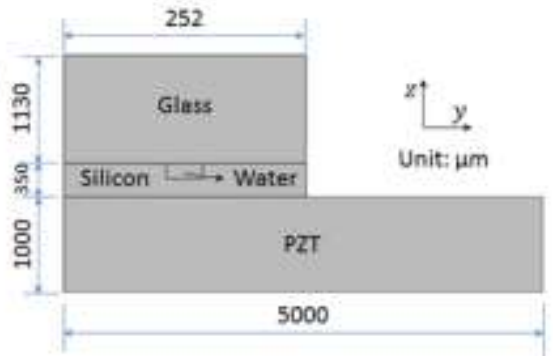

Fig. 2 (a) The 3D full model considered; (b) yz cross-section of (a).

Fig. 2(a) shows the 3D model considered, which represents a short section of the device investigated by Muller et al. $^{23}$. A schematic of different layers of the model is shown in Fig. 2(b), composed of a transducer layer (PZT), a matching layer (silicon), a fluid layer (water), and a reflector layer (glass). The model parameters are summarised in Table 1, including particle properties used in particle trajectory simulations. In order to balance the numerical accuracy and the computational load, a uniform distribution of swept mesh with an element size of $50 \mu \mathrm{m}$ in the fluid channel was used for the results presented here unless otherwise stated, which is chosen based on the mesh dependency study presented in ${ }^{9}$ which shows that $8 \sim 10$ elements within each acoustic wavelength is enough for the simulation of acoustic and streaming fields.

Table 1 3D Rayleigh streaming and particle trajectory model parameters

\begin{tabular}{|rl|rl|}
\hline Fluid volume $(x \times y \times z):$ & $1 \times 0.377 \times 0.157 \mathrm{~mm}^{3}$ & Excitation: & $40 \mathrm{~V}_{\mathrm{pp}}$ \\
\hline Driving frequency, $f:$ & $1.936 \mathrm{MHz}$ & Fluid density, $\rho_{f}:$ & $999.62 \mathrm{~kg} / \mathrm{m}^{3}$ \\
\hline Acoustic speed in fluid, $c_{f}:$ & $1481.4 \mathrm{~m} / \mathrm{s}$ & Dynamic viscosity of water, $\mu:$ & $1.0093 \times 10^{-3} \mathrm{~Pa} \mathrm{~s}$ \\
\hline Particle diameter, $d:$ & $0.5 \mu \mathrm{m} \mathrm{\&} 5 \mu \mathrm{m}$ & Acoustic speed in particle, $c_{p}:$ & $1962 \mathrm{~m} / \mathrm{s}$ \\
\hline Particle density, $\rho_{p}:$ & $1055 \mathrm{~kg} / \mathrm{m}^{3}$ & Mesh size: & $5 \times 10^{-5} \mathrm{~m}$ \\
\hline
\end{tabular}

The left and right walls $(x= \pm 0.5 \mathrm{~mm}$ ) were considered as plane wave radiation boundary conditions and the remaining walls as hard boundaries. The resonant frequency was found at $1.963 \mathrm{MHz}$ by using a parametric sweep to find the average acoustic energy density in the fluid layer versus driving frequency (the resonance was taken as the maximum of this function). The simulated acoustic pressure field is shown in Fig. 3. It can be seen that a lateral half-wavelength ( $y$ direction) standing wave field is generated in the fluid channel in this device and the acoustic pressure magnitude decreases from the centre $(x=0)$ to the left and right boundaries $(x= \pm 0.5 \mathrm{~mm})$ as energy traveling down the channel is largely absorbed by the tubing and connectors at the left and right ends. 
(a)

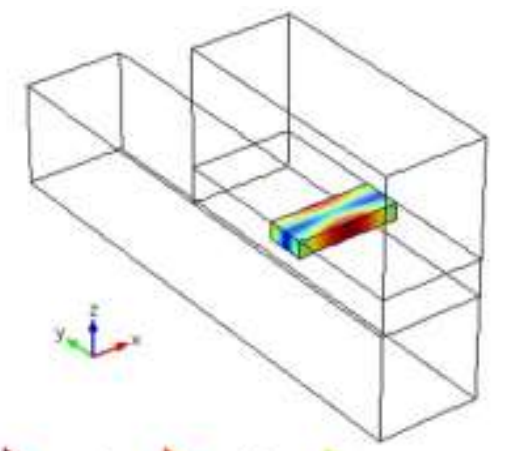

(b)

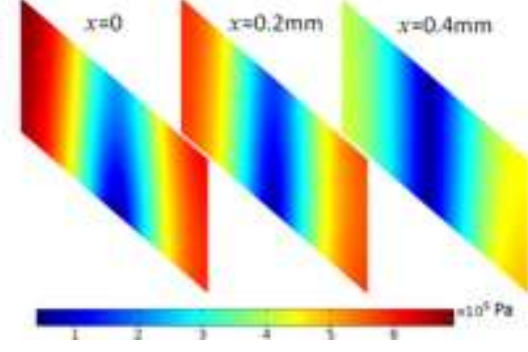

Fig. 3 (a) 3D acoustic pressure field within the fluid volume; (b) acoustic pressure magnitude on three vertical $y z$ planes.

In the creeping flow step of the method, the top and bottom walls of the fluid channel were considered as limiting velocity boundary conditions while the remaining four walls were considered as slip boundary conditions. Fig. 4 (a) shows the modelled acoustic streaming velocity magnitude on the surfaces of fluid channel. Fig. 4 (b) shows the $3 \mathrm{D}$ acoustic streaming field through three $y z$ planes, $x=0, x=0.2 \mathrm{~mm}$, and $x=0.4 \mathrm{~mm}$. The four counter-rotating vortices that can be seen within the lateral half-wavelength resonator are characteristic of classical Rayleigh streaming. Due to the acoustic variation along the channel axis ( $x$-direction), the magnitude of the streaming velocity is at a maximum at the centre $(x=0)$ of the device and decreases with distance from the centre because the acoustic energy density is strongest at the centre $(x=0)$ of the model.

(a)

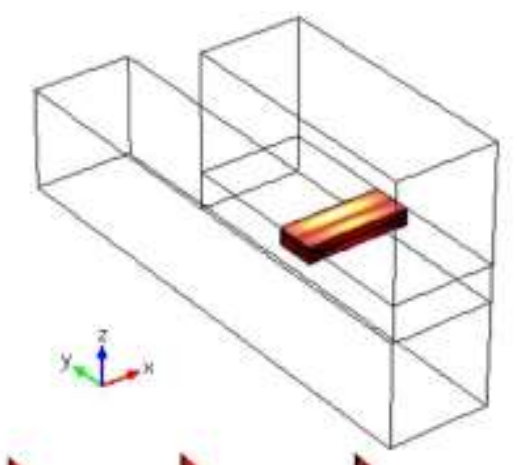

(b)

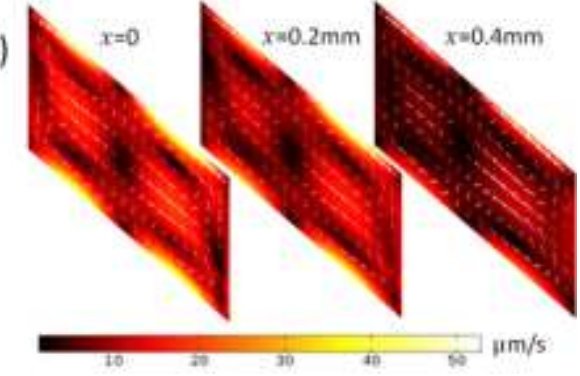

Fig. 4 (a) 3D acoustic streaming field within the fluid volume; (b) acoustic streaming field on three vertical yz planes.

In order to understand the effects of acoustic streaming on acoustophoretic motion of microparticles and compare with the experimental visualisation, a numerical simulation of particle trajectories is presented here. Neglecting the gravity force and buoyancy force, the movement of the particle within a standing wave field is determined by the combination of acoustic radiation force $(\mathrm{ARF})^{26}, \mathrm{~F}_{\mathrm{ac}}$, inertia, and the viscous drag on the particle, $F_{d}$ (sometimes called the acoustic streaming force, ASF, when the drag is caused by streaming motion). 


$$
\begin{gathered}
\frac{d}{d t}\left(m_{p} v\right)=F_{d}+F_{a c}, \\
F_{a c}=-\nabla\left(V_{0}\left(\frac{3\left(\rho_{p}-\rho_{f}\right)}{2 \rho_{p}+\rho_{f}} \overline{E_{k l n}}-\left(1-\frac{\beta_{p}}{\beta_{f}}\right) \overline{E_{p o t}}\right)\right), \\
F_{d}=6 \mu \pi r(u-v),
\end{gathered}
$$

where $m_{p}$ is the particle mass, $v$ is the velocity of the particle, $u$ is the fluid velocity, $\mu$ is the fluid viscosity, $r$ is the particle radius, $\overline{E_{k l n}}$ and $\overline{E_{p o t}}$ are the time average kinematic and potential energy, $\rho_{p}$ and $\rho_{f}$ are respectively the density of particle and fluid, $\beta_{p}$ and $\beta_{f}$ are the compressibility of particle and fluid, and $V_{0}$ is the particle volume.

The COMSOL 'Particle Tracing for Fluid Flow' module is used to implement these equations to simulate the particle trajectories. In order to compare with the experimental investigations shown in ${ }^{23}$, the trajectories of $0.5 \mu \mathrm{m}$ and $5 \mu \mathrm{m}$ particles are demonstrated here. Both ARF and ASF act on the tracer particles (polystyrene beads of diameter $0.5 \mu \mathrm{m}$ and $5 \mu \mathrm{m}$ ), resulting in the motion shown in Fig. 5 . It can be seen that the movements of 0.5 $\mu \mathrm{m}$ particles are dominated by the ASF as the pattern the particle trajectories form is closely related to the acoustic streaming field. However, $5 \mu \mathrm{m}$ particles are firstly driven to the pressure nodal plane by ARF and then slowly dragged to the up and bottom boundaries by ASF. A comparison between numerical simulation and experiments will be shown in the following discussion section.

(a)

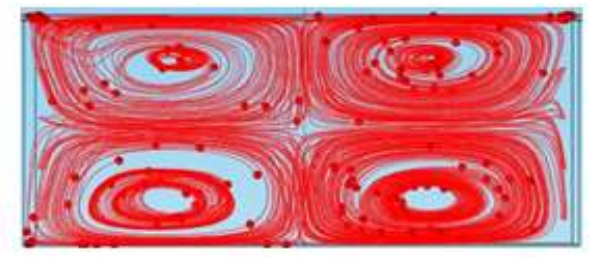

(b)

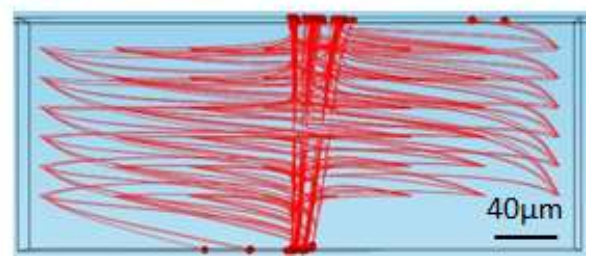

Fig. 5 Overall views along the channel axis ( $x$-direction) of modelled trajectories of $0.5 \mu \mathrm{m}$ particles (a) and $5 \mu \mathrm{m}$ particles (b), initially arranged in a $7 \times 8 \times 6$ array, where the spheres present the particles and the lines show respectively their trajectories.

In addition to the dominant Rayleigh streaming pattern in the $y z$ plane, the streaming also has components along the channel axis ( $x$ direction), which can cause particle migration along the channel, and is seen most clearly in the animation attached as a supplementary file. It can also be seen in Fig. 6 where the in-plane streaming velocity magnitude (Fig. 6 (a)) can be compared to the smaller but significant out-of-plane component along the channel axis. This exists due to the presence of acoustic energy gradients along the fluid channel ( $x$-direction). 
(a)

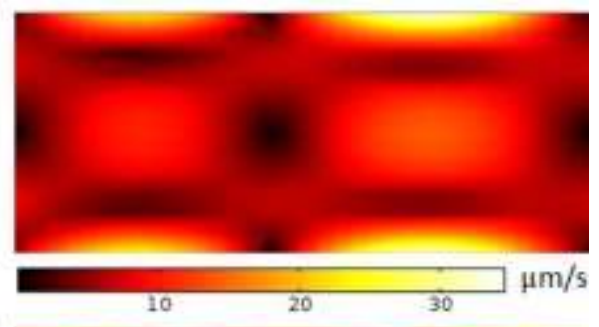

(b)

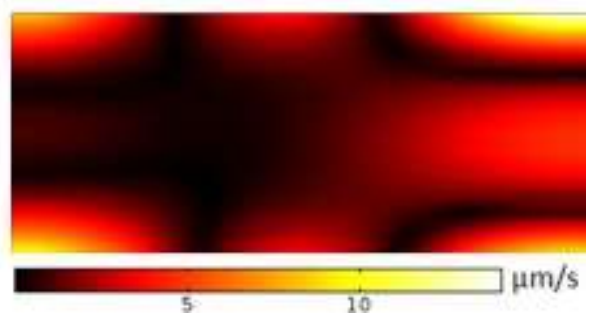

Fig. 6 A comparison of the magnitude of acoustic streaming velocity components on a yz plane $(x=-0.3 \mathrm{~mm}$, corresponding to the animation in the supplementary material): (a) In plane components; (b) out of plane component, (along channel).

\section{Discussion}

The acoustic streaming pattern obtained from numerical and experimental investigations can be compared from the trajectories of $0.5 \mu \mathrm{m}$ particles, which are dominated by the ASF. It can be seen from Fig. 5 (a) and Fig. 4 (b) in ${ }^{23}$ that classical Rayleigh streaming pattern is obtained from both methods within this lateral half-wavelength resonator.

Due to the quadratic dependence of the limiting velocity on the linear acoustic quantities, the relationship between the maximum streaming velocity in the device, $u_{2 \max }$, and the maximum acoustic pressure, $p_{\max }$, is expected to take the form

$$
u_{2 \max }=\alpha p_{\max }^{2}
$$

where $\alpha$ is a constant. On the other hand, the relationship between acoustic energy density and acoustic pressure can take the form

$$
E=\beta p^{2},
$$

where $\beta$ is a constant, so the comparison between experiment and model on the magnitude of acoustic streaming velocity can be achieved from the comparison of relationship between energy density and maximum streaming velocity

$$
u_{2 \max }=\frac{\alpha E_{\max }}{\beta}=\gamma E_{\max }
$$

Experimental work by Muller et al. ${ }^{23}$ found that when the energy density measured in the device is approximately $E_{\max }=(65 \pm 2) \mathrm{J} / \mathrm{m}^{3}$, the corresponding maximum streaming velocity (velocity of $0.5 \mu \mathrm{m}$ particles) measured is $\left(u_{2}\right)_{\max }=63 \mu \mathrm{m} / \mathrm{s}$. Therefore, the measured constant $\gamma$ presented in eqn. (12) is: $\gamma=(0.97 \pm 0.03) \times 10^{-6} \mathrm{~m}^{4} \mathrm{~J}^{-1} \mathrm{~s}^{-1}$.

In the model presented here, it is found that when $E_{\max }=54.8 \mathrm{~J} / \mathrm{m}^{3}$ then $\left(u_{2}\right)_{\max }=52.7 \mu \mathrm{m} / \mathrm{s}$. Therefore, the constant $\gamma$ of the model is: $\gamma=0.96 \times 10^{-6} \mathrm{~m}^{4} \mathrm{~J}^{-1} \mathrm{~s}^{-1}$.

It can be seen that the magnitude of the acoustic streaming velocities in the model and experiment are also in good agreement. 


\section{Investigation of an unusual vortex pattern}

\section{Background}

This section introduces a device presented in 2007 by Hagsater et al. ${ }^{13}$ and seeks to explain the unusual streaming pattern observed. Fig. 7 shows the chip configuration and the observed in-plane acoustic streaming pattern. It can be seen from Fig. 7 (b) that a $6 \times 6$ in-plane vortex pattern was generated. However, from both the measured trajectories of $5 \mu \mathrm{m}$ tracer particles (Fig. 4 (a) of ${ }^{13}$ ) and the simulated acoustic pressure Eigen mode (Fig. 4(c) of ${ }^{13}$ ), we can see a pattern of $6 \times 6$ antinodes in the square area of the fluid chamber, which would normally be expected to result in a $12 \times 12$ vortex pattern ( 2 vortices within each half wavelength for the classical Rayleigh streaming pattern). Therefore, the in-plane vortex pattern cannot be explained by classical Rayleigh streaming theory. In order to provide better understanding of this streaming pattern, a finite element model is presented here to simulate the $3 \mathrm{D}$ acoustic streaming field in this device and to investigate its origin.

(a)

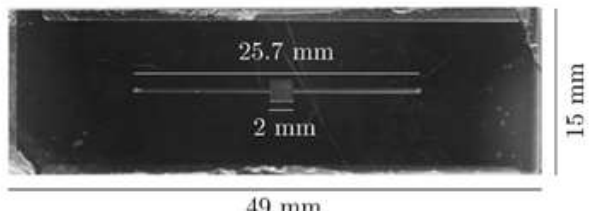

(b)

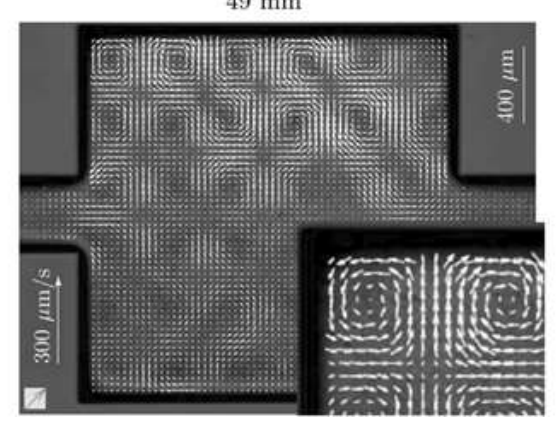

Fig. 7 Experimental investigation of Hagsater et al. ${ }^{13}$, where inset shows detail at top-left corner of chamber. Adapted from Ref. 13.
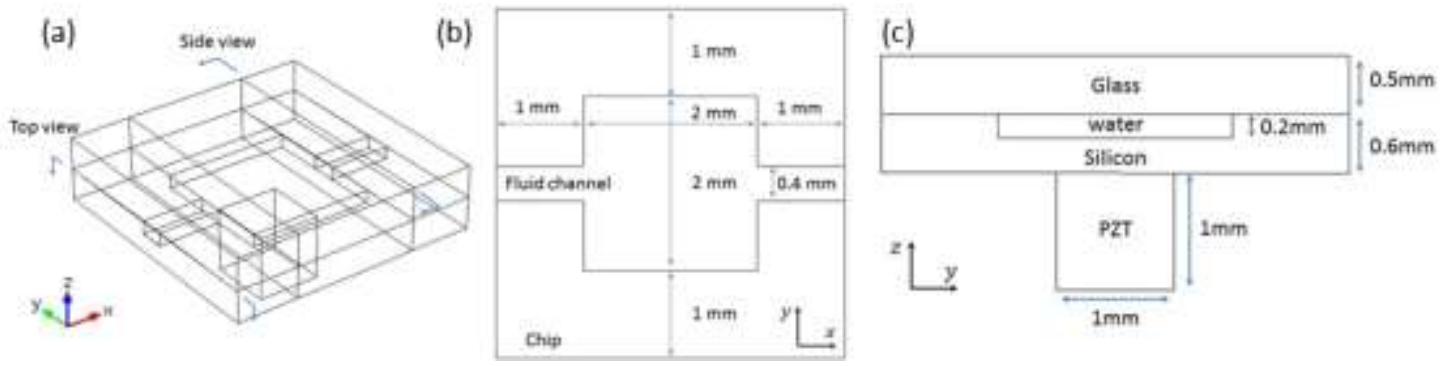

Fig. 8 (a) 3D full model; (b) top view; (c) side view.

\section{Finite Element model and Results}

Fig. 8 shows the schematic of our model, where (a) is a 3D view of the full device and (b) \& (c) are respectively top \& side views of the model with dimensioning. The origin of the coordinates was set at the centre of the interface between water and glass. All model parameters are summarised in Table 2.

Table 2 Model parameters

\begin{tabular}{|rl|rl|}
\hline Central square area $(x \times y \times z):$ & $2 \times 2 \times 0.2 \mathrm{~mm}^{3}$ & Excitation: & $40 \mathrm{~V}_{\mathrm{pp}}$ \\
\hline Driving frequency, $f:$ & $2.17 \mathrm{MHz}$ & Fluid density, $\rho_{f}:$ & $999.62 \mathrm{~kg} / \mathrm{m}^{3}$ \\
\hline Acoustic speed in fluid, $c_{f}:$ & $1481.4 \mathrm{~m} / \mathrm{s}$ & Dynamic viscosity of water, $\mu:$ & $1.0093 \times 10^{-3} \mathrm{~Pa} \mathrm{~s}$ \\
\hline
\end{tabular}

Firstly the mesh, as with the previous model, was chosen based on the mesh dependency study presented in ${ }^{9}$ such that 8 10 elements within each acoustic wavelength are enough for the simulation of acoustic and streaming fields. In order to balance the computational load and numerical accuracy, a mesh size of $0.08 \mathrm{~mm}$ was used for the results presented here, resulting in an estimated mesh-induced numerical error of $2 \%$. 
The resonant frequency was found at $2.193 \mathrm{MHz}$ by using a parametric sweep to find the maximum average acoustic energy density in the fluid layer versus driving frequency. The resonant frequency gives a 2D standing wave in the $x$ and $y$ directions in this shape of fluid channel. In order to match the experimental measurement, the results shown below were obtained from the model run at frequency of $2.17 \mathrm{MHz}$. A 3D view of the acoustic pressure field within the fluid channel is plotted, Fig. 9 (a). It can be seen that throughout the device the magnitude of acoustic pressure is almost constant along the $z$ axis. In the $x y$ plane, in the central square area of fluid channel ( $2 \mathrm{~mm} \times 2 \mathrm{~mm}$ ), a primary standing wave field (close to three wavelengths in extent, Fig. 9 (b)) is established in the $y$ direction and in the $x$ direction the acoustic pressure distribution also shows a standing wave field of three wavelengths due to plane wave radiation boundaries on two ends of fluid channel.

(a)

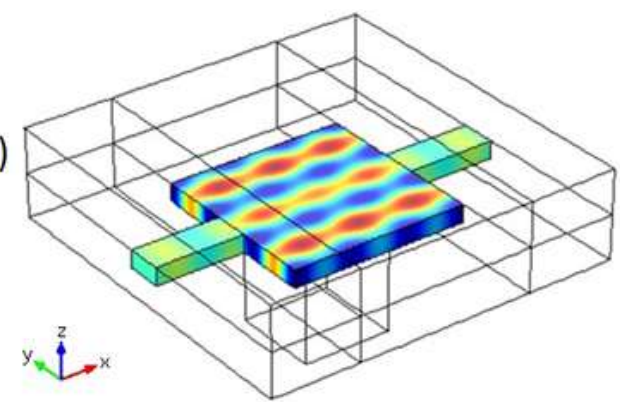

(b)

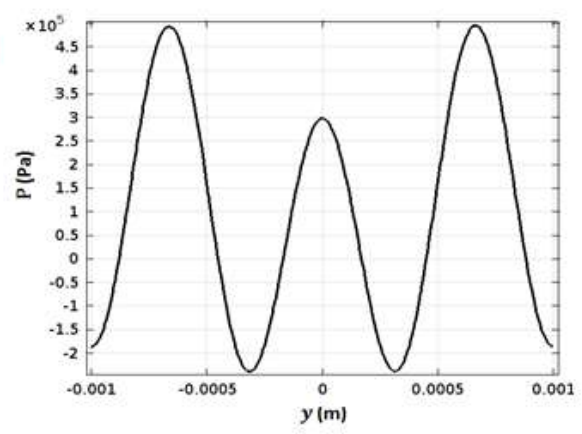

Fig. 9 Simulated acoustic pressure field: (a) A 3D view; (b) Magnitude of acoustic pressure along the central line of fluid channel $(x=0,-1 \mathrm{~mm} \leq y \leq 1 \mathrm{~mm}, z=0)$.

In the creeping flow step of the method, the top and bottom boundaries ( $z=0$ and $z=-0.02 \mathrm{~mm}$ ) of fluid channel were considered as limiting velocity boundary conditions while the other walls were slip boundary conditions. In order to help visualise the acoustic 3D streaming field, streaming in both the $y z$ cross-section $(x=0.5 \mathrm{~mm})$ and $x z$ cross-section $(y=0.5 \mathrm{~mm})$ in the central square area of fluid channel are plotted in Fig. 10. Due to a dominant standing wave being established ( $3 \lambda)$ in the $y$ direction and the shape of fluid channel, a classical Rayleigh streaming vortex pattern is observed in the $y z$ cross section, Fig. 10 (a). Note that although the chamber is square, the entry and exit channels in the $x$ direction mean that the field is not symmetrical. A similar but weaker vortex pattern is seen in the $z x$ plane, Fig. 10 (b), which is the Rayleigh streaming from the weaker $x$-directed standing wave. 

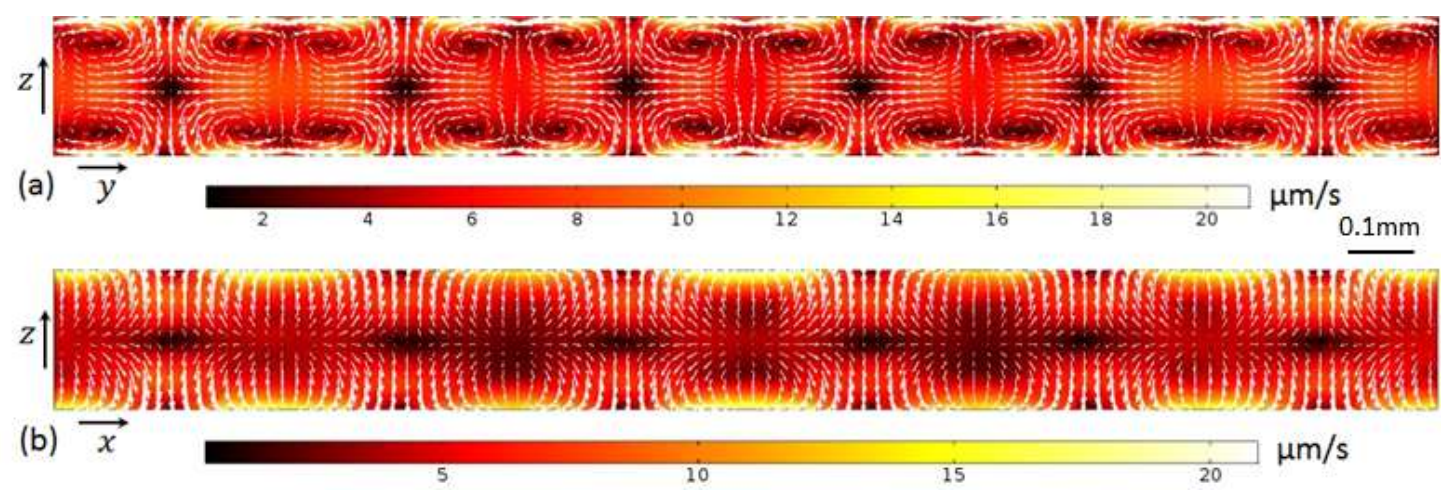

Fig. 10 Acoustic streaming field on (a) a $y z$ cross-section at $x=-0.5 \mathrm{~mm}$, and (b) a $x z$ cross-section at $y=-0.5 \mathrm{~mm}$. The arrows show the orientation of acoustic streaming field and colour bars plot the magnitude of acoustic streaming velocities.

In order to compare modelled results to the experimentally observed in-plane vortex pattern, a top view of the acoustic streaming field in the central square area of fluid channel is plotted. Fig. 11 (a) shows the streaming field at the mid height (plane $z=-0.1 \mathrm{~mm}$ ) and Fig. 11 (b) shows the streaming pattern at plane just below the very top of the fluid channel (plane $z=-0.04 \mathrm{~mm}$ ). The reason for choosing this plane to present the in-plane acoustic streaming pattern is that the direction of Rayleigh streaming velocities on this plane is mainly perpendicular to the $x y$ plane, which can be seen from Fig. 10 (a), so the in-plane vortex pattern can be seen more clearly. In this $x y$ plane a $6 x 6$ vortex pattern in the square fluid channel is obtained, which compares well with the experimental visualisation of Hagsater et al. ${ }^{13}$. However, the orientation of acoustic streaming in each single vortex is opposite to the experimental visualisation. Similarly, another $6 \times 6$ in-plane vortex pattern can be seen on the plane $z=-0.16 \mathrm{~mm}$.

In order to investigate the behaviour of this in-plane acoustic streaming pattern in more detail, the model was also run at frequencies around the reported driving frequency. It was found that at all frequencies the $6 \times 6$ in-plane vortex pattern was observed on the same planes. In addition, another two models (included as supplementary information) were considered with a change in the $x$ and $y$ dimensions of the channel to $1.95 \times 1.95 \mathrm{~mm}^{2}$ (model 3) and $2.05 \times 2.05 \mathrm{~mm}^{2}$ (model 4) to investigate the sensitivity of this in-plane streaming pattern to the size of the fluid chamber. It was found that in model 3, both the 6 x 6 vortex pattern and the Rayleigh streaming pattern was close to the results presented here. In model 4, the Rayleigh streaming pattern is similar to the model presented here but the $6 \times 6$ in-plane vortex pattern has the direction of rotation of each vortex opposite to that shown in Fig. 11 (b) (i.e. the same as that reported in the experimental visualisation). The differences of orientation in each vortex in model 4 and the results presented in this paper are believed to be related to the change of direction of the active sound intensity field in these two models, which will be analysed in more detail in the discussion below. 


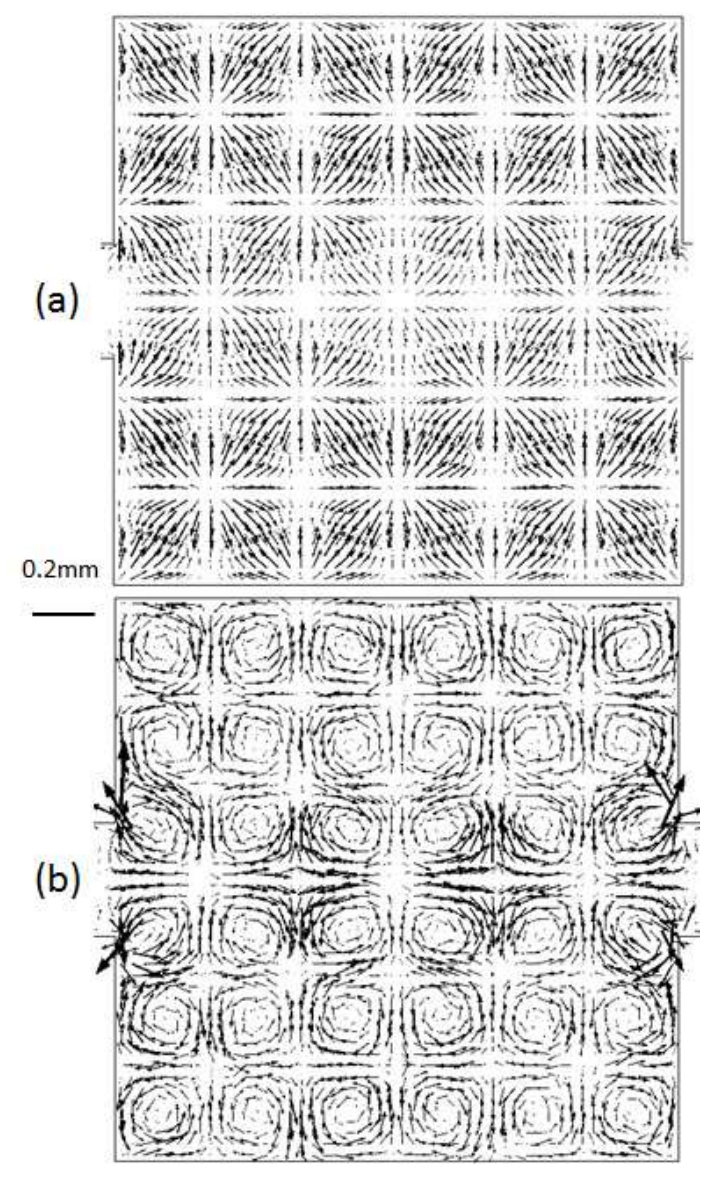

Fig. 11 (a) Modelled acoustic streaming field (a) on plane $z=-0.1 \mathrm{~mm}$; (b) on the plane $z=-0.04 \mathrm{~mm}$.

\section{Discussion}

We have previously analysed the in-plane (i.e. parallel to the transducer face) streaming patterns in a planar half wave resonator. In that case we found that a $2 \times 2$ vortex pattern was obtained regardless of the multiple wavelengths in the $x$ and $y$ directions. For that device the following approximations held:

$$
\frac{d u_{1}}{d x} \ll \frac{d w_{1}}{d z} \text { and } \frac{d v_{1}}{d y} \ll \frac{d w_{1}}{d z}
$$

Under these assumptions the limiting velocity shown in Eqns. (3)-(4) can be approximated to ${ }^{9}$

$$
\begin{aligned}
& u_{L} \approx-\frac{1}{4 \omega} \operatorname{Re}\left(-2 i u_{1}^{*} \frac{d w_{1}}{d z}\right), \\
& v_{L} \approx-\frac{1}{4 \omega} \operatorname{Re}\left(-2 i v_{1}^{*} \frac{d w_{1}}{d z}\right),
\end{aligned}
$$

which (taking $u_{L}$ as an example) can also be expressed using the acoustic intensity, $C$,

$$
\begin{gathered}
C_{x}=\frac{1}{2} u_{1}^{*} p, \\
u_{L} \approx \frac{1}{\rho_{0} c^{2}} \operatorname{Re}\left(C_{x}\right) .
\end{gathered}
$$

Please note an error in our previous paper': Eqn.(19) was presented there with a minus before the expression for $u_{L}$ (a result of a sign error in Eqn. (21) of that paper). This error does not change the results and conclusions of that paper as the modelling there was performed with the full expression for limiting velocity (found in equations 6 and 7 of that paper). 
In this device, where the two orthogonal standing waves along $x$ and $y$ are the dominant and which has negligible standing wave in the $\mathrm{z}$ direction, a different set of approximations are valid:

$$
\frac{d w_{1}}{d z} \ll \frac{d u_{1}}{d x} \text { and } \frac{d w_{1}}{d z} \ll \frac{d v_{1}}{d y}
$$

In this case, Eqns. (3)-(4) can now be approximated as

$$
\begin{aligned}
& u_{L} \approx-\frac{1}{4 \omega} \operatorname{Re}\left\{q_{x}+u_{1}^{*}\left[(2+i)\left(\frac{d u_{1}}{d x}+\frac{d v_{1}}{d y}\right)\right]\right\}, \\
& v_{L} \approx-\frac{1}{4 \omega} \operatorname{Re}\left\{q_{y}+v_{1}^{*}\left[(2+i)\left(\frac{d u_{1}}{d x}+\frac{d v_{1}}{d y}\right)\right]\right\} .
\end{aligned}
$$

Using Eqn. (20) we can write the complex pressure as $^{27}$

$$
p \approx \frac{i \rho_{0} c^{2}}{\omega}\left(\frac{d u_{1}}{d x}+\frac{d v_{1}}{d y}\right)
$$

Thus Eqns. (21)-(22) can be expressed in terms of the active sound intensity (the real part) and reactive sound intensity (the imaginary part of complex intensity):

$$
\begin{aligned}
& u_{L}=-\frac{1}{4 \omega} \operatorname{Re}\left(q_{x}\right)-\frac{1}{\rho_{0} c^{2}} \operatorname{Im}\left(C_{x}\right)-\frac{1}{2 \rho_{0} c^{2}} \operatorname{Re}\left(C_{x}\right), \\
& v_{L}=-\frac{1}{4 \omega} \operatorname{Re}\left(q_{y}\right)-\frac{1}{\rho_{0} c^{2}} \operatorname{Im}\left(C_{y}\right)-\frac{1}{2 \rho_{0} c^{2}} \operatorname{Re}\left(C_{y}\right) .
\end{aligned}
$$

In order to distinguish the terms that drive the Rayleigh type streaming patterns found in Fig. 10 (a) from those which produce the in-plane vortex pattern (Fig. 11 (b)), we must establish which of the driving terms have rotation in the $x y$ plane (at the boundary where the limiting velocity is calculated, $z=0$ ).

Firstly, the contribution of the first terms, $q_{x}$ and $q_{y}$. In the linear (inviscid) acoustic approximation the acoustic particle velocity, $\boldsymbol{u}$, is irrotational: $:^{27}$

$$
\nabla \times \boldsymbol{u}=0 .
$$

Using this relation (along with the fact that the spatial derivatives of $\nabla \times \boldsymbol{u}$ must also be zero, we find that the curl of the field $\boldsymbol{Q}=\left(q_{x}, q_{y}, 0\right)$ is everywhere zero and hence will not contribute to the $x y$ plane 6 x6 vortex pattern.

Then, the contribution of the remaining terms. As discussed in our previous analysis ${ }^{9}$, according to Fahy ${ }^{28}$, only the active intensity, the real part of complex sound intensity can have a rotational component in a standing wave field and this rotation reflects the elliptical path that fluid particles take rather than circulation of energy on a larger scale.

Thus the rotational component of the streaming field in the $x y$ plane is proportional to the active sound intensity components of Eqns. (24)-(25). The active sound intensity is plotted in Fig. 12 and can be seen to closely resemble the rotational part of the modelled and experimental fields found in Fig. 11 (b) and Fig. 7 (b). Interestingly the rotation of the limiting velocity is in the opposite direction to that of the active intensity under this approximation (Eqn.(20)), compared to that previously investigated where the approximations of Eqn.(15) were valid.

We are now in a position to understand why the direction of $x y$ plane rotation is different in this model and model 4. Examining the models we find what the change of dimension of fluid chamber changes the relative phases of the standing waves in the $x$ and $y$ directions, which in turn changes the direction of rotation of the active sound intensity field, and hence streaming field. 


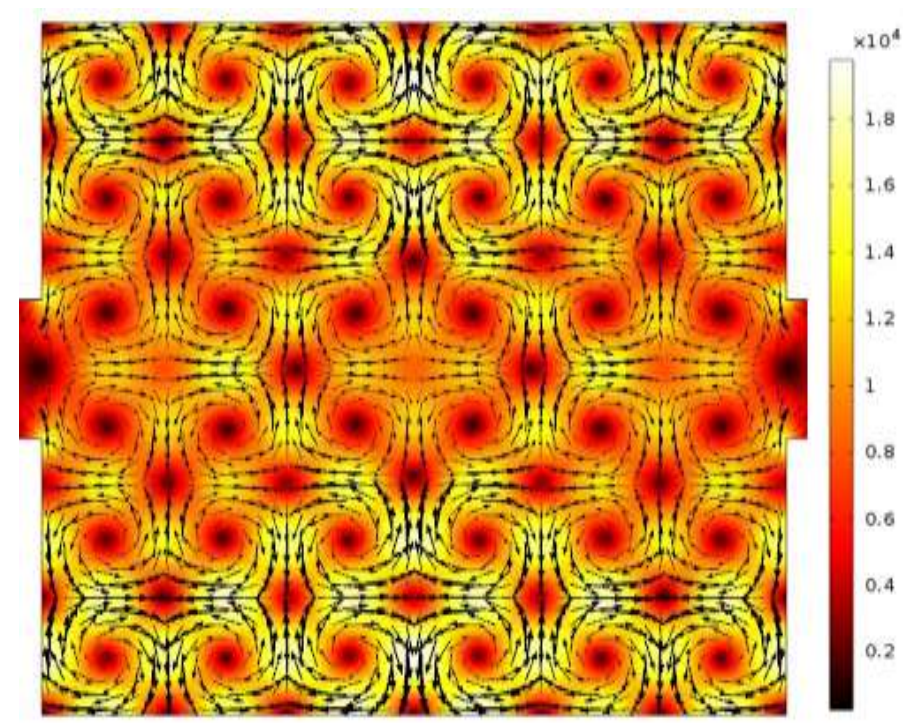

Fig. 12 Modelled active sound intensity field $\left(\mathrm{W} / \mathrm{m}^{2}\right)$ on a limiting velocity boundary in the main fluid channel.

\section{Conclusions}

The 3D Rayleigh streaming pattern in an acoustofluidic device has been simulated using the limiting velocity method and its effects on acoustphoretic motion of microparticles are presented. While results from 2D simulations of streaming in uniform channels can show good accuracy, this 3D method permits modelling of subtle effects relating to non-uniformities and resonances in the length direction of channels, and also the modelling of more complex structures, suggesting that streaming motion exists in all three directions. The simulated acoustic streaming field compared well with the experimental investigations.

Additionally, acoustic streaming due to two orthogonal standing waves in a square device was numerically simulated and its mechanism considered. Previous experimental work had reported a regular array of vortices that could not be explained by analogy with Rayleigh streaming since the periodicity of the structure did not match such a hypothesis. We find that in certain planes our model predicts similar circulatory patterns to those found in the experiments, which was found to be closely related to the active sound intensity field. With a slight change on the size of the fluid channel, the direction of orientation of in-plane streaming pattern was changed due to the change of active sound intensity field although the Rayleigh streaming pattern remained the same. Further experimental verification that the pattern found in the model is consistent with that observed is necessary to consider the origin of these vortices solved, however the mechanism described here would seem a strong candidate.

As illustrated, numerical results obtained from this computationally efficient method can not only represent 3D acoustic and streaming fields in real acousto-microfluidic devices but also provide good comparisons with experimental measurements. This should allow such models to be used to predict the streaming fields in microfluidic devices to provide optimization of device designs. This limiting velocity method is valid for modelling boundary induced streaming fields when the local curvatures of the boundaries are large in comparison to the viscous penetration depth and the streaming velocities are low enough to be within a strictly laminar regime. It does not, however, model Eckart type streaming ${ }^{8,29}$ induced by bulk absorption of sound, which can be modelled as a volume force on the fluid ${ }^{30,31}$. Thus this method is not suitable for modelling the majority of streaming found in high frequency surface acoustic wave systems ${ }^{32,33}$, but it would be interesting to explore to what extent boundary driven streaming contributes in these systems.

\section{Acknowledgements}

The authors gratefully acknowledge the financial support for this work received from China Scholarship Council (CSC) and the EPSRC under the Sonotweezers project (EP/G012075/1). 


\section{References}

1. L. Rayleigh, Phil. Trans., 1883, 175, 1-21.

2. P. J. Westervelt, J. Acoust. Soc. Am., 1952, 25, 60-67.

3. W. L. Nyborg, J. Acoust. Soc. Am., 1953, 25, 68-75.

4. H. Schlichting, Physikalische Zeitschrift, 1932, 33, 327-335.

5. S. Boluriaan and P. J. Morris, Aeroacoustics, 2003, 2 (3\&4), 255-292.

6. M. Wiklund, R. Green and M. Ohlin, Lab Chip, 2012, 12, 2438-2451.

7. M. F. Hamilton, Y. A. Ilinskii and E. A. Zabolotskaya, J Acoust Soc Am, 2003, 113, 153160.

8. C. Eckart, Phys. Rev., 1947, 73, 68-76.

9. J. Lei, P. Glynne-Jones and M. Hill, Lab Chip, 2013, 13, 2133-2143.

10. P. Glynne-Jones, C. E. M. Demore, C. W. Ye, Y. Q. Qiu, S. Cochran and M. Hill, IEEE T Ultrason Ferr, 2012, 59, 1258-1266.

11. M. W. Thompson and A. A. Atchley, J Acoust Soc Am, 2005, 117, 1828-1838.

12. M. Nabavi, M. H. K. Siddiqui and J. Dargahi, Meas Sci Technol, 2007, 18, 1811-1817.

13. S. M. Hagsater, T. G. Jensen, H. Bruus and J. P. Kutter, Lab Chip, 2007, 7, 1336-1344.

14. R. Barnkob, P. Augustsson, T. Laurell and H. Bruus, Lab Chip, 2010, 10, 563-570.

15. M. Koklu, A. C. Sabuncu and A. Beskok, J Colloid Interf Sci, 2010, 351, 407-414.

16. M. Rossi, R. Segura, C. Cierpka and C. J. Kahler, Exp. Fluids, 2012, 52, 1063-1075.

17. C. Cierpka, R. Segura, R. Hain and C. J. Kahler, Meas Sci Technol, 2010, 21, 1-13.

18. C. Cierpka, M. Rossi, R. Segura and C. J. Kahler, Meas Sci Technol, 2011, 22, 1-10.

19. C. Cierpka, M. Rossi, R. Segura, F. Mastrangelo and C. J. Kahler, Exp. Fluids, 2012, 52, 605-615.

20. M. Kawahashi and M. Arakawa, JSME International Journal Series B-Fluids and Thermal Engineering, 1996, 39, 280-286.

21. M. K. Aktas and B. Farouk, J Acoust Soc Am, 2004, 116, 2822-2831.

22. P. B. Muller, R. Barnkob, M. J. H. Jensen and H. Bruus, Lab Chip, 2012, 12, 4617-4627.

23. P. B. Muller, M. Rossi, Á. G. Marín, R. Barnkob, P. Augustsson, T. Laurell, C. J. Kähler and H. Bruus, Phys Rev E, 2013, 88, 023006.

24. C. P. Lee and T. G. Wang, J. Acoust. Soc. Am., 1989, 85, 1081-1088.

25. Comsol Multiphysics 4.3, http://www.comsol.com/.

26. H. Bruus, Lab Chip, 2012, 12, 1014-1021.

27. L. E. Kinsler, Fundamentals of acoustics, Wiley, New York, 2000.

28. F.J.Fahy, Sound intensity, E \& FN Spon, London, 1995.

29. S. S. Sadhal, Lab Chip, 2012, 12, 2292-2300.

30. A. L. Bernassau, P. Glynne-Jones, F. Gesellchen, M. Riehle, M. Hill and D. R. S. Cumming, Ultrasonics, 2014, 54, 268-274.

31. D. Möller, T. Hilsdorf, J. Wang and J. Dual, AIP Conference Proceedings, 2012, 1433, 775-778.

32. X. Ding, P. Li, S.-C. S. Lin, Z. S. Stratton, N. Nama, F. Guo, D. Slotcavage, X. Mao, J. Shi, F. Costanzo and T. J. Huang, Lab Chip, 2013, 13, 3626-3649.

33. S. Li, X. Ding, F. Guo, Y. Chen, M. I. Lapsley, S.-C. S. Lin, L. Wang, J. P. McCoy, C. E. Cameron and T. J. Huang, Analytical Chemistry, 2013, 85, 5468-5474. 